\title{
Genome wide association study of fatty acid composition in Duroc swine
}

\author{
Vanessa S. Viterbo ${ }^{1,2}$, Bryan Irvine M. Lopez ${ }^{1}$, Hyunsung Kang ${ }^{1}$, Hoonseop Kim¹, \\ Choul-won Song ${ }^{1}$, and Kang Seok Seo,*
}

\footnotetext{
* Corresponding Author: Kang Seok Seo Tel: +82-61-750-3231, Fax: +82-61-750-3230 E-mail: sks@sunchon.ac.kr
}

${ }^{1}$ Department of Animal Science and Technology, Sunchon National University, Suncheon 57922, Korea 2 Department of Animal Science, College of Agriculture, Central Luzon State University, Science City of Muńoz, 3120, Philippines

ORCID

Vanessa S. Viterbo

https://orcid.org/0000-0001-7549-2028

Bryan Irvine M. Lopez

https://orcid.org/0000-0002-3288-5849

Hyunsung Kang

https://orcid.org/0000-0001-6309-0857

Hoonseop Kim

https://orcid.org/0000-0002-2066-5283

Choul-won Song

https://orcid.org/0000-0001-8207-748X

Kang Seok Seo

https://orcid.org/0000-0002-6116-6114

Submitted Oct 20, 2017; Revised Dec 22, 2017; Accepted Jan 30, 2018
Objective: Genome wide association study was conducted to identify and validate candidate genes associated with fatty acid composition of pork.

Methods: A total of 480 purebreed Duroc pigs were genotyped using IlluminaPorcine60k bead chips while the association test was implemented following genome-wide rapid association using Mixed Model and Regression-Genomic Control (GRAMMAR-GC) approach. Results: A total of 25,29, and 16 single nucleotide polymorphisms (SNPs) were significantly associated with stearic (18:0), oleic (18:1) and saturated fatty acids (SFA), respectively. Genome wide significant variants were located on the same region of swine chromosome 14 (SSC14) that spanned from 120 to $124 \mathrm{Mb}$. Top SNP ALGA008191 was located at $5 \mathrm{~kb}$ near the stearoylCoA desaturase $(S C D)$ gene. This gene is directly involved in desaturation of stearic acid into oleic acid. General relationship of significant SNPs showed high linkage disequilibrium thus genome-wide signals was attributed to $S C D$ gene. However, understanding the role of other genes like elongation of very long chain fatty acids-3 (ELOVL3) located on this chromosomal segment might help in further understanding of metabolism and biosynthesis of fatty acids. Conclusion: Overall, this study provides evidence that validates $S C D$ gene as strong candidate gene associated with fatty acid composition in Duroc pigs. Moreover, this study confirms significant SNPs near ELOVL3 gene.

Keywords: Duroc; Fatty Acid; Genome Wide Association Study (GWAS); Stearoyl-CoA Desaturase $(S C D)$ Gene

\section{INTRODUCTION}

Quality of lipids, fats and fatty acid compositions are important factors that influence both the organoleptic quality and nutritive value of meat. Fatty acid composition showed strong correlation with meat firmness and oiliness. These traits are believed to be associated with the rate of meat oxidation after slaughtering and during meat processing [1]. Investigation of fatty acid composition in Duroc pigs showed that saturated fatty acid (SFA) and monounsaturated fatty acids (MUFA) were highly heritable with genetic estimates of 0.50 to 0.57 while polyunsaturated fatty acids (PUFA) moderately with 0.25 to 0.46 [2]. These heritability estimates showed strong potential for improvement of meat quality in pigs. To date, inclusion of fatty acids in genomic breeding selection has been limited; however, with the robust advancement of technology to identify detailed quantitative trait loci (QTL), the importance of fatty acid to meat quality improvement will be soon elucidated.

Genome-wide association studies (GWAS) use high-throughput genotyping technologies such as Illumina60K bead chip to assay hundreds of thousands of single nucleotide polymorphism (SNP) and to associate them with diseases or quantitative traits [3]. Implementation of GWAS has resulted in the discovery of many genes directly involved in a 
variety of disease related traits, genes associated with economically important traits, and genes involved in many metabolic and physiological activities within cells and tissues. The cost of genotyping and complex analysis of data are the challenges researchers are facing today in performing GWAS, with low replications using independent populations and lower sample size leading to erroneous results. Thus, this study was conducted to identify genetic variants associated with fatty acid and to provide verification on previously reported significant QTL and identify novel genes influencing fatty acid composition in Duroc pigs.

\section{MATERIALS AND METHODS}

\section{Animals and phenotypic data}

A total of 480 purebred female Duroc pigs were selected for fatty acid composition analysis. Animals were raised under commercial conditions and fed ad libitum during the entire fattening period. After reaching 120 to $130 \mathrm{~kg}$, pigs were slaughtered in batches following the standards set by the Korea Institute for Animal Production Quality Evaluation (KAPE).

Tissues from longissimus dorsi muscles were collected immediately after slaughter and kept frozen at $-20^{\circ} \mathrm{C}$. The total lipids were quantified following a protocol conducted by previous studies [4]. Briefly, extracted fats were saponified with potassium hydrate-ethanol solution and then methyl sterified using boron triflouride-methanol solution. Individual fatty acid was subsequently determined quantitatively by gas chromatography (Model 6890, Hewlett-Packard Co., Wilmington, DE, USA). The temperature of the inlet was $180^{\circ} \mathrm{C}$, the oven was warmed from $180^{\circ} \mathrm{C}$ to $200^{\circ} \mathrm{C}$ for 25 minutes, and the temperature of the detector sensor was $260^{\circ} \mathrm{C}$. Nitrogen gas was used as the carrier, and an omega wax $320(30 \mathrm{~m} \times 0.32 \mathrm{~mm}$ $\times 0.25 \mu \mathrm{m}$, Supelco, Bellefonte, PA, USA) was used as capillary column.

\section{Genotyping and genomic quality control}

Genomic DNA was extracted from ear tissue of each animal using the phenol/chloroform method. Four hundred eighty experimental pigs were genotyped for 61,565 SNPs using Illumina PorcineSNP60K bead chips according to the manufacturer's protocols. Quality control measures were implemented using threshold levels: genotyping call rate per individual and per marker $>90 \%$, minor allele frequency $>0.05$, and divergence from Hardy-Weinberg equilibrium (HWE) with p-value of 0.001. Individuals and SNP markers that did not conform to the threshold levels were eliminated prior to the association test using PLINK v. 1.09 [5].

\section{Statistical analysis}

Genome-wide association analysis was conducted following genome-wide rapid association using a mixed model and regression-genomic control (GRAMMAR-GC) approach [6,7]. Fatty acid composition was corrected for fixed effects and polygenic covariation effects based on the following model:

$$
\gamma_{1}=\mu+\sum_{j} \beta_{j} c_{i j}+G_{i}+e_{i}
$$

Where, $\gamma_{1}$ is the phenotype of the ith individual, $c_{i j}$ is the value of the $j$ th covariate or fixed effect for the individual $i, \beta_{j}$ is an estimate of the jth fixed effect or covariate, and $G_{i}$ and $e_{i}$ are random additive polygenic and residual effects, respectively. Pedigrees of the animals were retrieved back up to second generation and explored for possible relatedness and were used to create numerator relationship matrix. Fixed effects consisted of four farms and slaughter batches, while the covariate was the slaughter age. Commercial farms consist of Nunghyop, Samsung, Wonsan, and Chuksan while slaughtering was done on 37 batches. Based on this model, derivation of residuals was conducted using the blupf90 programs [8]. Residuals were then used as dependent traits in a simple linear regression analysis for each SNP marker.

$$
\hat{\mathrm{e}}_{\mathrm{i}}=\mu+\mathrm{kg}_{\mathrm{i}}+\mathrm{e}
$$

Where, $\mu$ is the mean, $\mathrm{g}$ is the vector of genotypes, $\mathrm{k}$ is the regression coefficient and $\mathrm{e}$ is the vector of random residuals. The empirical p-values based on Wald statistics were adjusted using the genomic control (GC) method by estimation of the inflation factor $\left(\lambda_{\mathrm{CG}}\right)$ based on median chi-square statistics. Corrected population stratifications were illustrated in the quantile-quantile plot constructed within the $R$ environment. The genome-wide significant threshold was determined based on Bonferroni adjustment at a significance of 0.05 (p-values $=1.29 \times 10^{-6}$ ).

The linkage disequilibrium coefficient $\left(\mathrm{r}^{2}\right)$ was calculated between SNP markers located in the same chromosomal segment that showed significant association with fatty acid composition. Haploblocks were constructed and visualized using Haploview v.4.2 [9].

SNP and candidate gene annotation were retrieved using Ensembl annotation of Sscrofa 10.2 genome version in NCBI data base [10] while functional classification of candidate genes with the target chromosomal segment was investigated using DAVID online annotation tools [11].

\section{RESULTS AND DISCUSSION}

\section{Phenotype and genotype}

The descriptive statistics of the fatty acid composition of longissimus dorsi muscles are presented in Table 1. Fatty acid composition was dominated by oleic acid (18:1) with $41.83 \%$ 
Table 1. Summary statistics for fatty acid composition in Duroc

\begin{tabular}{lcccccc}
\hline Fatty acid & No. & Mean & SD & Min & Max & $\mathbf{h}^{2}$ \\
\hline Palmitic (16:0) & 471 & 25.46 & 1.06 & 22.66 & 28.29 & 0.50 \\
Palmitoleic (16:1) & 291 & 3.17 & 0.58 & 1.9 & 4.63 & 0.36 \\
Stearic (18:0) & 451 & 13.19 & 1.15 & 10.13 & 16.07 & 0.17 \\
oleic (18:1) & 469 & 41.83 & 2.33 & 35.45 & 47.41 & 0.34 \\
Linoleic (18:2) & 451 & 8.49 & 1.76 & 4.61 & 12.8 & 0.18 \\
Linolenic (18:3) & 386 & 0.3 & 0.16 & 0.03 & 0.66 & 0.18 \\
SFA & 282 & 41.58 & 1.71 & 37.22 & 46.19 & - \\
MUFA & 285 & 47.94 & 2.05 & 47.94 & 52.65 & - \\
PUFA & 289 & 10.49 & 2.17 & 5.55 & 16.56 & - \\
\hline
\end{tabular}

SD, standard deviation; $h^{2}$, heritability estimates; SFA, saturated fatty acid; MUFA, monounsaturated fatty acid; PUFA, polyunsaturated fatty acid.

which are comparable with previous investigations [12]. In this study, ten genotyped animals with less than $90 \%$ call rate, and 30,426 low quality SNP markers were eliminated prior to the association test. Most of these SNP markers were removed due to minor allele frequency of less than $5 \%$, with the total number of 20,928 SNP. Thus, a total of 470 genotyped animals and a subset of 39,919 SNPs were used in the association test. Number of low quality SNP in this study is slightly higher compared with previous studies [13]. Incidence of high number of low quality SNPs narrows possibility of false positive association, and at the same time limiting probabilities of a true positive association. This scenario might suggest the reason for low positive result in other GWAS test. Therefore, quality control in data handling and data collection improving high quality SNPs with strengthen genome-wide association results.

\section{Population stratification}

Population stratification was accounted by GC approach by correcting test statistics. The quantile-quantile (Q-Q) plot illustrates the observed chi-square statistics against expected chi-square statistics for association test of stearic (18:0), oleic
(18:1) and SFA shown in Figure 1. Results revealed that estimates of genomic inflation factor for stearic, oleic and SFA were $1.009,1.03$, and 0.995 , respectively. These values conclude that population stratification was effectively accounted in this study, thus relevant associations are not due to population stratification but instead to a true positive association.

\section{Association study}

In this study, genomic segment on same region of swine chromosome 14 (SSC14) about 120 to $124 \mathrm{Mb}$ showed significant association with stearic (18:0), oleic (18:1) and SFA. Details of significant SNPs are given in Table 2. These associations were visualized in the Manhattan plot illustrated in Figure 2. Strong SNPs for these traits were located on the same chromosomal region which implied that candidate gene on this region is involved in the desaturation process of stearic acid into oleic acid, thus total SFA is also altered. Desaturation of fatty acids or addition of double band in fatty acid chain is affected by a complex relationship of specific enzymes and by various factors. To date, intricate mechanism of fatty acid synthesis and metabolism is not yet fully understood. Moreover, conversion of stearic acid into oleic acid connotes negative correlations.

One candidate gene that is directly involved in fatty acid biosynthesis and lipid synthesis was identified. The top SNP ALGA0081091 was located at about $5 \mathrm{~kb}$ from the stearoylCoA desaturase (SCD) gene. The same chromosomal segment was previously identified; however candidate genes associated with fatty acid composition that are situated on the other chromosomes were not elucidated in this study $[14,15]$. Results showed that a high number of low quality SNPs reduces probability of detecting previously identified genes. Although a high number of SNPs was removed, the chromosomal segment where the $S C D$ gene was located remains significant.

The SCD gene is located at $120.9 \mathrm{Mb}$ on SSC14 with a total length of 16,231 base pairs (bp). This gene is responsible for desaturation of SFA by the addition of single double band
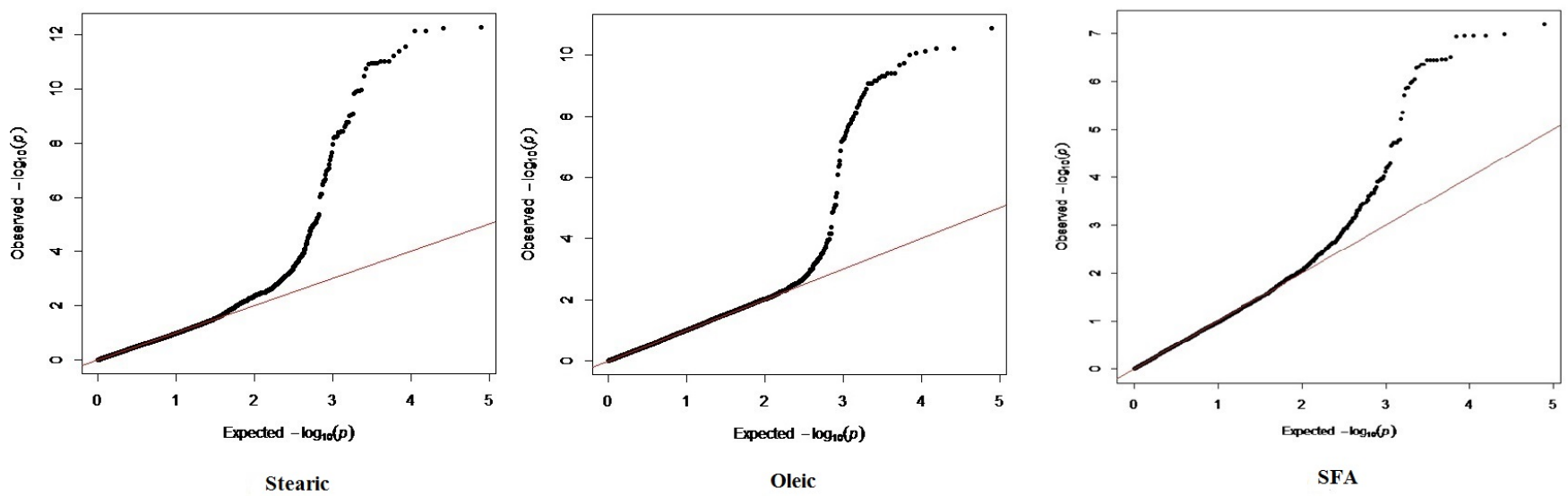

Figure 1. Quantile-quantile (Q-Q) plots of SNP markers in genome wide association study of fatty acid composition. A, B, and C represents stearic, oleic, and saturated fatty acid (SFA), respectively. 
Table 2. Genome-wide significant SNP markers for oleic, stearic and SFA

\begin{tabular}{|c|c|c|c|c|c|c|c|c|c|c|}
\hline \multirow{2}{*}{$\begin{array}{l}\text { CHR } \\
14\end{array}$} & \multirow{2}{*}{$\begin{array}{c}\text { SNP } \\
\text { ASGA0066177 }\end{array}$} & \multirow{2}{*}{$\begin{array}{r}\text { Position }^{1)} \\
123214577\end{array}$} & \multicolumn{2}{|c|}{ Allele } & \multirow{2}{*}{$\begin{array}{l}\text { MAF } \\
0.38\end{array}$} & \multirow{2}{*}{$\begin{array}{c}\text { Nearby gene } \\
\text { GBF1 }\end{array}$} & \multirow{2}{*}{$\begin{array}{c}\begin{array}{c}\text { Functional } \\
\text { consequence }\end{array} \\
\text { Intron }\end{array}$} & \multirow{2}{*}{$\frac{\text { p-value }^{*, 2)}}{7.67^{-11}}$} & \multirow{2}{*}{$\frac{p \text {-value }}{1.45^{-08}}$} & \multirow{2}{*}{$\frac{p \text {-value }}{{ }^{*, 4)}}$} \\
\hline & & & $A$ & $G$ & & & & & & \\
\hline 14 & ALGA0081091 & 120986865 & A & $C$ & 0.34 & $S C D$ & $5 \mathrm{~kb}$ & $8.64^{-11}$ & $7.96^{-10}$ & $2.99^{-07}$ \\
\hline 14 & DIAS0004744 & 123268607 & A & G & 0.38 & CUEDC2,NFKB2 & Intron & $2.20^{-10}$ & $1.41^{-08}$ & - \\
\hline 14 & ALGA0081164 & 122935134 & C & $A$ & 0.38 & HPS6 & Missense & $3.89^{-10}$ & $2.13^{-08}$ & - \\
\hline 14 & ALGA0081161 & 122861163 & G & $A$ & 0.38 & C14H10orf76 & Intron & $3.89^{-10}$ & $2.13^{-08}$ & - \\
\hline 14 & ASGA0066165 & 22819384 & G & $A$ & 0.38 & C14H10orf76 & Intron & $4.83^{-10}$ & $2.35^{-08}$ & - \\
\hline 14 & ASGA0066162 & 122718232 & G & $A$ & 0.38 & LOC100037948 & Intron & $4.83^{-10}$ & $2.35^{-08}$ & - \\
\hline 14 & ASGA0066174 & 123083682 & G & A & 0.38 & ELOVL3 & $3^{\prime}$ utr Variant & $6.74^{-10}$ & $3.31^{-08}$ & - \\
\hline 14 & ALGA0081097 & 121330920 & G & $A$ & 0.29 & & & $8.72^{-10}$ & $2.30^{-11}$ & $1.10^{-07}$ \\
\hline 14 & CASI0010164 & 121305916 & A & $C$ & 0.29 & & & $8.72^{-10}$ & $2.30^{-11}$ & $1.10^{-07}$ \\
\hline 14 & ASGA0066144 & 122072563 & A & G & 0.34 & & Intron & $3.21^{-09}$ & $1.02^{-11}$ & $5.25^{-07}$ \\
\hline 14 & H3GA0042098 & 122667984 & G & $A$ & 0.38 & & Intron & $5.06^{-09}$ & $5.61^{-08}$ & - \\
\hline 14 & ASGA0066158 & 122427131 & G & $A$ & 0.34 & FBXW4 & Intron & $7.86^{-09}$ & $2.85^{-11}$ & $1.34^{-06}$ \\
\hline 14 & INRA0046731 & 122279650 & A & G & 0.34 & $B T R C$ & Intron & $7.86^{-09}$ & $2.85^{-11}$ & $1.34^{-06}$ \\
\hline 14 & H3GA0042103 & 123318264 & G & $A$ & 0.35 & LOC102167427, TMEM180 & intron,upstream & $1.07^{-08}$ & $1.07^{-07}$ & - \\
\hline 14 & H3GA0042104 & 123339204 & A & $\mathrm{C}$ & 0.35 & ACTR1A & Intron & $1.31^{-08}$ & $1.85^{-07}$ & - \\
\hline 14 & INRA0046735 & 122482603 & A & G & 0.34 & FBXW4, LOC102162056 & intron,upstream & $1.65^{-08}$ & $2.63^{-11}$ & $1.40^{-06}$ \\
\hline 14 & INRA0046761 & 123475867 & G & A & 0.36 & SUFU & Intron & $1.90^{-08}$ & $6.62^{-08}$ & - \\
\hline 14 & H3GA0042111 & 123496150 & A & G & 0.3 & TRIM8 & Intron & $3.36^{-08}$ & $1.20^{-06}$ & - \\
\hline 14 & ASGA0066192 & 123409339 & A & G & 0.33 & SUFU & Intron & $4.50^{-08}$ & - & - \\
\hline 14 & ALGA0081147 & 122390223 & G & $A$ & 0.29 & FBXW4 & Intron & $5.59^{-08}$ & $4.52^{-10}$ & $3.47^{-07}$ \\
\hline 14 & ASGA0066098 & 120474440 & A & G & 0.28 & $A B C C 2$ & Intron & $6.17^{-08}$ & $1.37^{-09}$ & $6.32^{-08}$ \\
\hline 14 & ASGA0066137 & 122043297 & C & G & 0.29 & LOC102161206 & Intron & $6.59^{-08}$ & $3.48^{-09}$ & - \\
\hline 14 & ASGA0066126 & 121556717 & A & G & 0.3 & PAX2 & Intron & - & $5.81^{-11}$ & $1.03^{-07}$ \\
\hline 14 & ALGA0081112 & 121556717 & A & G & 0.26 & PAX2 & Intron & - & $4.77^{-09}$ & - \\
\hline 14 & CASI0007888 & 121556717 & A & G & 0.21 & HPSE2 & Intron & - & $2.47^{-08}$ & - \\
\hline 14 & ASGA0066024 & 121556717 & C & $A$ & 0.18 & LOC102162220 & Intron & - & $4.77^{-08}$ & - \\
\hline 14 & ALGA0081070 & 121556717 & A & G & 0.46 & DNMBP & Intron & - & $5.18^{-08}$ & - \\
\hline 14 & INRA0046585 & 121556717 & A & $\mathrm{C}$ & 0.18 & HOGA1 & Intron & - & $5.67^{-07}$ & - \\
\hline 14 & ASGA0066116 & 121467984 & G & $A$ & 0.34 & & & - & - & $3.48^{-07}$ \\
\hline 14 & ALGA00811117 & 121945296 & G & $A$ & 0.34 & & & - & - & $4.41^{-07}$ \\
\hline 14 & ASGA0066146 & 122124515 & G & $A$ & 0.29 & & & - & - & $4.92^{-07}$ \\
\hline 14 & ASGA0091963 & 118732842 & G & A & 0.29 & & & - & - & $1.01^{-06}$ \\
\hline
\end{tabular}

SNP, single nucleotide polymorphism; SFA, saturated fatty acid; MAF, minor allele frequency.

1) Position: SNP locations were derived in Sus scrofa 10.2 assembly.

2),3,4) $p$-value, $p$-values of significant SNP for oleic, stearic, and SFA, respectively.

* Bonferoni adjustment at $5 \%$ ( $p$ value $\left.1.026 \times 10^{-6}\right)$, at $1 \%\left(p 3.29 \times 10^{-7}\right)$.

between carbon 9 and 10 on the acyl chain and catalyzing the synthesis of oleic and palmitoleic acids. Oleic and palmitoleic acids are then utilized in the synthesis of various kinds of lipids, confirming the importance of SCD on biological properties of fatty acids and complex lipids [16,17]. Expression of $S C D$ gene at different levels in lipogenesis, lipolysis and fatty acid transport showed genetic variability of this gene between $\mathrm{Wu}$ jin and Landrace pigs [18]; A meta-analysis of GWAS on five populations that includes crossbreed of Duroc, Landrace and Yorkshire (DLY) showed strong evidence of association between SCD with palmitic, palmitoleic, stearic, and arachidonic acids [19]. In addition, haplotypes of $S C D$ gene showed strong influence on melting point of fatty acids in Duroc population [20].
Furthermore, SCD polymorphism is a relevant tool to increase MUFA and MUFA/PUFA ratio of dry cured hams in crossbreed Duroc and Iberian pigs [13]. In this study, allele A at ALGA0081091 SNP increases oleic acid while decreasing stearic acid which compliments the elongation of stearic acid to oleic acid by SCD enzyme. Therefore, this study validates SCD as a strong candidate gene influencing SFA and MUFA in Duroc pigs.

Alternatively, the signal detected in this study spanned from $120 \mathrm{Mb}$ up to $124 \mathrm{Mb}$. This chromosomal segment harbors other genes involved in lipid synthesis. The SNP ASGA0066174 is located at downstream untranslated variant of elongation of very long chain fatty acids-3 (ELOVL3) gene. This gene interacts with various co-factors, controlling synthesis of sat- 

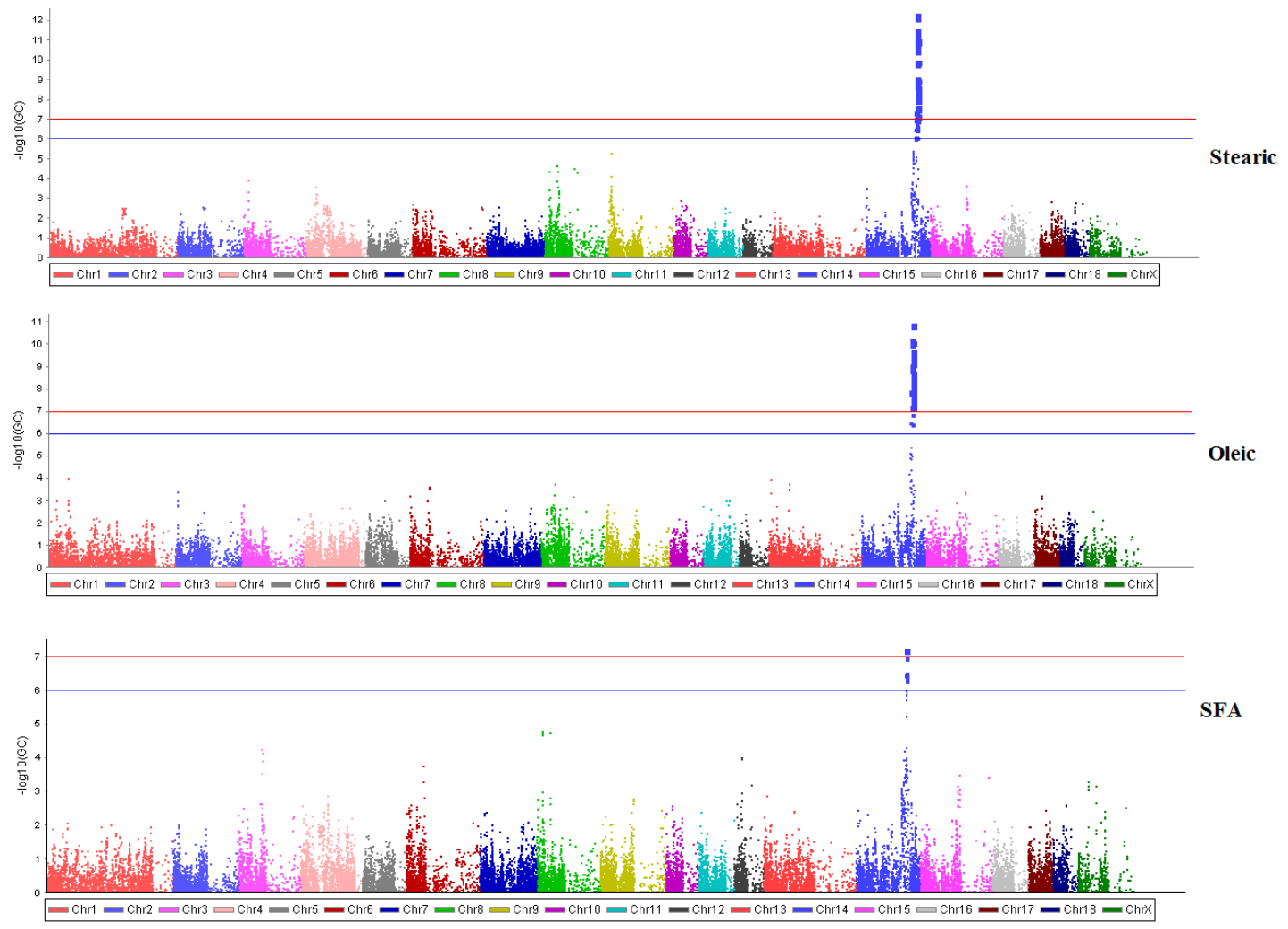

Figure 2. Manhattan plots of genome wide association study (GWAS) for stearic (A), oleic (B) and saturated fatty acid (SFA) (C). Chromosomes are represented on the $\mathrm{X}$-axis while $\mathrm{p}$-values are on the $\mathrm{Y}$-axis. Bonferroni threshold $\mathrm{p}$-values at $5 \%$ and $1 \%$ are indicated by the blue and red horizontal lines, respectively.

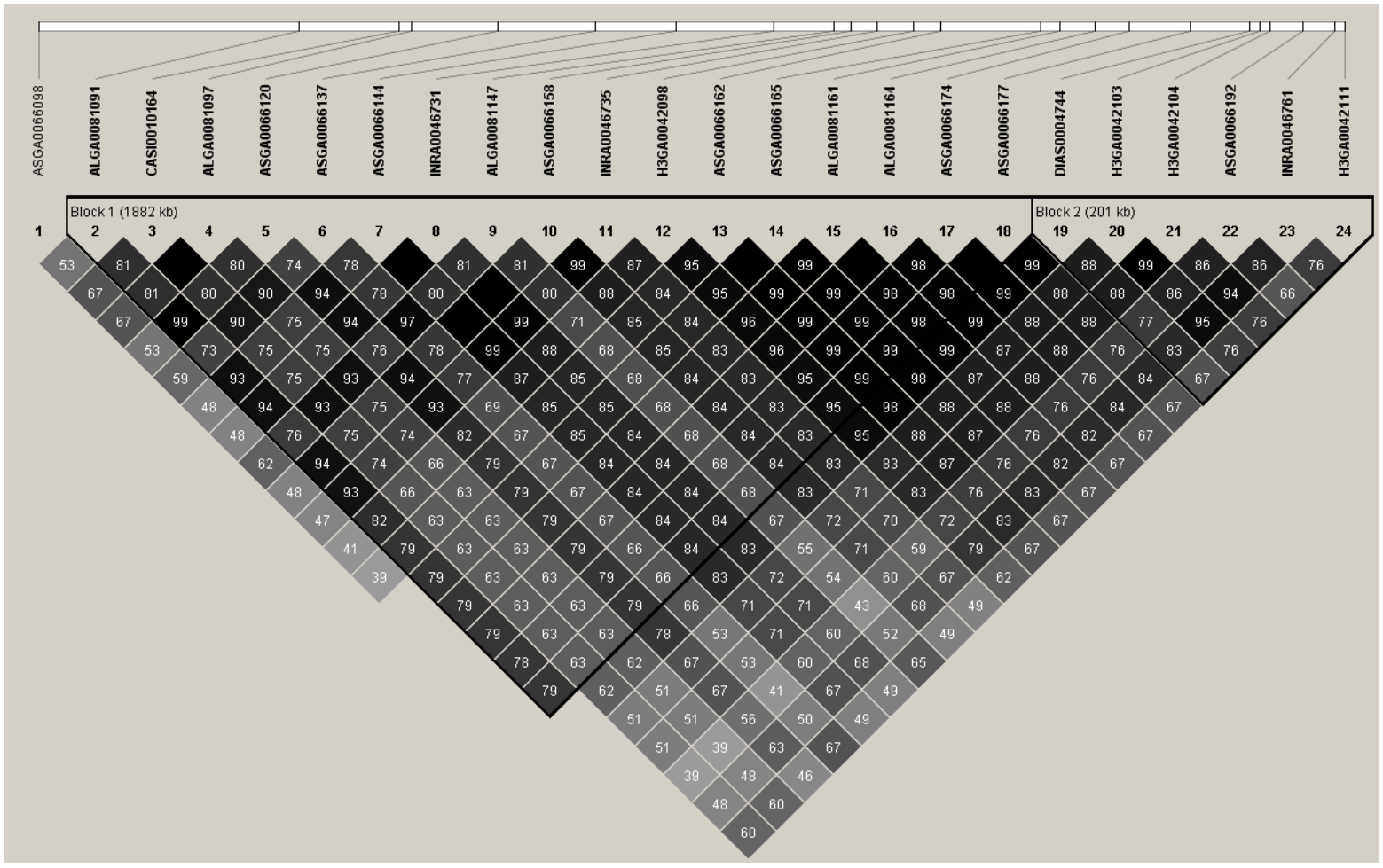

Figure 3. Linkage disequilibrium $\left(r^{2}\right)$ diagram of single nucleotide polymorphism (SNP) markers in chromosome 14 associated with fatty acids. The numbers outside the blocks represent SNP order in this region and the numbers inside the blocks indicate linkage disequilibrium (LD) coefficients $\left(r^{2}\right)$. The SNP grouped in each triangle box are grouped in one block based on LD information. The red mark indicates the position of stearoyl-CoA desaturase (SCD) gene. 
urated and MUFAs in specific tissues [21,16]. Investigation of the phenotypic diversity that arises from intensive genomic selection identified a highly differentiated region in chromosome 14 at about 123.08 to $123.41 \mathrm{Mb}$ that is unique in Duroc swine, where the ELOVL3 is located [22]. Moreover, peroxisome proliferator-activated receptor gamma gene, a candidate gene that influences fatty acid composition in swine, through the coregulation with ELOVL3 and very long chain fatty acid induces adipogenesis in mouse adipocytic 3T3-L1 cells, suggesting relevant participation of ELOVL3 in biosynthesis of lipids [23]. Conversely, general relationship of significant SNP (Figure 3) illustrates high linkage disequilibrium between significant SNP proposing coincident association between fatty acids and ELOVL3 since ASGA0066174 is not the top SNP, while very limited studies support the significant influence of ELOVL3 in fatty acid composition of pork.

Recently, the same chromosomal segment was reported in a GWAS in Duroc pigs which agrees not to discriminate candidate causative SNP around SCD gene $[24,19]$. Strong linkage disequilibrium between significant SNP limits the evidence that ELOVL3 is a strong candidate gene in fatty acid manipulation, but this does not eliminate the possible importance ELOVL3 in fatty acid manipulation, which requires further investigation.

\section{CONCLUSION}

Results identified a significant QTL on SSC14 (120 to $124 \mathrm{Mb}$ ) associated with stearic, oleic and SFA in Duroc pigs. The candidate gene located in this chromosomal segment is the $S C D$ gene that has a direct influence on fatty acid composition and has been identified in previous studies. Located on the same chromosomal segment is ELOVL3. Hence, this information provides relevant evidence for $S C D$ gene as a strong candidate gene in the improvement of fatty acid in Duroc pigs.

\section{CONFLICT OF INTEREST}

We certify that there is no conflict of interest with any financial organization regarding the material discussed in the manuscript.

\section{ACKNOWLEDGMENTS}

This work was carried out with the support of "Cooperative Research Program for Agriculture Science and Technology Development" (Project No. PJ012704012018) Rural Development Administration, Republic of Korea.

\section{REFERENCES}

1. Wood J, Enser M, Fisher A, et al. Fat deposition, fatty acid composition and meat quality: a review. Meat Sci 2008;78: 343-58.

2. Gjerlaug-Enger E, Aass L, Odegard J, Kongsro J, Vangen O. Genetic parameter of fat quality in pigs measured by nearinfrared spectroscopy. Animal 2011;5:1495-505.

3. Pearson TA, Manolio TA. How to interpret a genome-wide association study. JAMA 2008;299:1335-44.

4. Folch J, Lees M, Sloane-Stanley G. A simple method for the isolation and purification of total lipids from animal tissues. J Biol Chem 1957;226:497-509.

5. Purcell S, Neale B, Todd-Brown K, et al. PLINK: a tool set for whole-genome association and population-based linkage analyses. Am J Hum Genet 2007;81:559-75.

6. Aulchenko YS, De Koning D-J, Haley C. Genomewide rapid association using mixed model and regression: a fast and simple method for genomewide pedigree-based quantitative trait loci association analysis. Genetics 2007;177:577-85.

7. Amin N, Van Duijn CM, Aulchenko YS. A genomic background based method for association analysis in related individuals. PloS One 2007;2:e1274.

8. Aguilar I, Misztal I, Tsuruta S, Legarra A, Wang H. PREGSF90POSTGSF90: computational tools for the implementation of single-step genomic selection and genome-wide association with ungenotyped individuals in BLUPF90 programs. Proceedings 10th World Congress Genetics Applied Livestock Production; 2014.

9. Barrett J, Fry B, Maller J, Daly M. Haploview: analysis and visualization of $\mathrm{LD}$ and haplotype maps. Bioinformatics 2005; 21:263-5.

10. National Center for Biotechnology Information (NCBI) [Internet]. Bethesda, MD, USA: National Library of Medicine (US), National Center for Biotechnology Information; 1988 [cited 2017 Oct 20]. Available from: http://www.ncbi.nlm.nih.gov

11.Jiao X, Sherman BT, Huang DW, et al. DAVID-WS: a stateful web service to facilitate gene/protein list analysis. Bioinformatics 2012;28:1805-6.

12.Klensporf-Pawlik D, Szydlowski M, Kaczmarek A, et al. The fatty acid composition of the Longissimus dorsi muscle, subcutaneous and visceral fats differ in four commercial pig breeds. J Anim Feed Sci 2012;21:661-76.

13. Uemoto Y, Nakano H, Kikuchi T, et al. Fine mapping of porcine SSC14 QTL and SCD gene effects on fatty acid composition and melting point of fat in a Duroc purebred population. Anim Genet 2012;43:225-8.

14. Yang B, Zhang W, Zhang Z, et al. Genome-wide association analyses for fatty acid composition in porcine muscle and abdominal fat tissues. PloS One 2013;8:e65554.

15. Muñoz M, Rodríguez MC, Alves E, et al. Genome-wide analysis of porcine backfat and intramuscular fat fatty acid composition using high-density genotyping and expression data. BMC Genomics 2013;14:845.

16. Guillou H, Zadravec D, Martin PG, Jacobsson A. The key roles 
of elongases and desaturases in mammalian fatty acid metabolism: Insights from transgenic mice. Prog Lipid Res 2010; 49:186-99.

17. Miyazaki M, Ntambi JM. Role of stearoyl-coenzyme A desaturase in lipid metabolism. Prostaglandins Leukot Essent Fatty Acids 2003;68:113-21.

18.Zhao S, Ren L, Chen L, et al. Differential expression of lipid metabolism related genes in porcine muscle tissue leading to different intramuscular fat deposition. Lipids 2009;44:102937.

19.Zhang W, Zhang J, Cui L, et al. Genetic architecture of fatty acid composition in the longissimus dorsi muscle revealed by genome-wide association studies on diverse pig populations. Genet Sel Evol 2016;48:5.

20. Henriquez-Rodriguez E, Tor M, Pena R, Estany J. A polymorphism in the stearoyl-CoA desaturase gene promoter increases monounsaturated fatty acid content in dry-cured ham. Meat Sci 2015;106:38-43.

21.Jakobsson A, Westerberg R, Jacobsson A. Fatty acid elongases in mammals: their regulation and roles in metabolism. Prog Lipid Res 2006;45:237-49.

22. Wilkinson S, Lu ZH, Megens H-J, et al. Signatures of diversifying selection in European pig breeds. PLoS Genet 2013;9: e1003453.

23. Kobayashi T, Fujimori K. Very long-chain-fatty acids enhance adipogenesis through coregulation of Elovl3 and PPAR $\gamma$ in 3T3-L1 cells. Am J Physiol Endocrinol Metab 2012;302:E1461E71.

24. Ros-Freixedes R, Gol S, Pena RN, et al. Genome-wide association study singles out $S C D$ and $L E P R$ as the two main loci influencing intramuscular fat content and fatty acid composition in Duroc pigs. PloS One 2016;11:e0152496. 